\title{
A demonstration that electroweak theory can violate parity automatically (leptonic case)
}

\author{
C. Furey \\ Cavendish Laboratory, JJ Thomson ave, Cambridge, CB3 OHE, UK \\ Department of Applied Mathematics and Theoretical Physics, \\ Centre for Mathematical Sciences, Wilberforce road, Cambridge, CB3 OWA, UK \\ nf252@cam.ac.uk
}

Received 9 November 2017

Revised 10 January 2018

Accepted 15 January 2018

Published 10 February 2018

\begin{abstract}
We bring to light an electroweak model which has been reappearing in the literature under various guises. ${ }^{1-5}$ In this model, weak isospin is shown to act automatically on states of only a single chirality (left). This is achieved by building the model exclusively from the raising and lowering operators of the Clifford algebra $\mathbb{C l}(4)$. That is, states constructed from these ladder operators mimic the behaviour of left- and right-handed electrons and neutrinos under unitary ladder operator symmetry. This ladder operator symmetry is found to be generated uniquely by $s u(2)_{L}$ and $u(1)_{Y}$. Crucially, the model demonstrates how parity can be maximally violated, without the usual step of introducing extra gauge and extra Higgs bosons, or ad hoc projectors.
\end{abstract}

Keywords: Electroweak; parity violation; Clifford algebra; leptons; ladder operators; unitary symmetry; $\mathrm{U}(2)$; supersymmetry; spinor; $\mathrm{Cl}(4)$; number operator; weak hypercharge; chiral; standard model of particle physics.

PACS numbers: 02.10.Hh, 02.10.Ud, 02.20.Qs, 02.90.+p, 11.30.Er, 11.30.Rd, 12.15.-y, 14.6.St

\section{Introduction}

Violation of parity in nature was a finding that few could have possibly foreseen. When Lee and Yang proposed parity violation in their Nobel prize winning paper, $\frac{6}{6}$ they noted that up until then, parity symmetry in the weak interactions had largely been assumed without question. Furthermore, when Wu subsequently discovered this parity violation experimentally in the decay of Cobalt- $60, \frac{7}{}$ the result

This is an Open Access article published by World Scientific Publishing Company. It is distributed under the terms of the Creative Commons Attribution 4.0 (CC-BY) License. Further distribution of this work is permitted, provided the original work is properly cited. 
was met with immediate skepticism. ${ }^{8}$ However, her discovery withstood repeated verification. 9,10

As a direct result of such experiments, modern electroweak theory is now built on the acknowledgement that weak isospin violates parity. Furthermore, the violation is understood to be maximal: weak isospin acts only on those fermions which are left-handed.

Now, the idea that $S U(2)_{L}$ acts on only left-handed fermions can seem puzzling and arbitrary. That is, why would nature not treat left and right equally?

Indeed, since as early as the time of Lee and Yang,,$\frac{6}{}$ researchers have set up models where parity can be restored, in some way. More recent models suggest that this left-right symmetry could at least be restored at high energies. ${ }^{11}-15$

However, as Senjanović pointed out, $\frac{13}{2}$ this restoration often comes at a price. For example, the introduction of an $S U(2)_{R}$ symmetry, to complement $S U(2)_{L}$, carries new gauge bosons into the theory. Furthermore, new Higgs fields are then subsequently introduced, in order to spontaneously break the symmetry back down to that which we observe.

After 60 years, this topic is no less engaging than it ever was, as is clear by the amount of recent progress in models constructed to address the issue. $\frac{15-21}{2}$

In this review, we expose an answer to this long-standing riddle, which has appeared and reappeared in the literature. ${ }^{1-5}$ Remarkably, this solution does not require the introduction of extra gauge or Higgs bosons. (Although this is not to say that these results would necessarily be in conflict with those models which do.)

In the late 1990s, P. Woit proposed such an electroweak model, originating from supersymmetric quantum mechanics. $\frac{1}{}$ Here, the electroweak group $U(2)=$ $S U(2)_{L} \times U(1)_{Y} / \mathbb{Z}_{2}$ resulted as the subgroup of $S O(4)$ frame rotations which preserves a required complex structure. In the earlier papers of Barducci, Buccella, Casalbuoni, Gatto, Lusanna, and Sorace, $\stackrel{2-4}{-}$ a related mathematical structure was identified in the context of tensor products of Clifford algebras (although its physical interpretation varied). More recently, this same construct appeared once again, this time arising from the use of the normed division algebras over the real numbers. $\frac{5,22}{}$ Although these models originate remarkably from different perspectives, they share a common mathematical theme. Without appealing to supersymmetry nor division algebras, here we will describe in detail the core idea on which these models converge.

In this paper, we will present this core model, built on $\mathbb{C l}(4)$ Clifford algebraic ladder operators. These ladder operators are taken to transform under the unitary symmetry that preserves their structure. We show that this ladder symmetry is generated uniquely by $s u(2)$ and $u(1)$. Next, we demonstrate how these $s u(2)$ generators act nontrivially on only half of the model's states, which we consequently identify as a left-handed doublet, complemented by a pair of right-handed singlets. Our $u(1)$ generator automatically gives charges consistent with weak hypercharge. In short, the model yields quite efficiently the basic electroweak behaviour for one generation of standard model leptons, together with a right-handed neutrino. 
This result may be useful for authors with an interest in left-right asymmetry models,,$\underline{6-21}$ existing Clifford algebraic particle models, $\underline{23}-\underline{34}$ beyond-the-standardmodel proposals,,$\underline{35}-\underline{37}$ and noncommutative geometry. $\underline{38-44}$

The aim of this review paper is to bring to a wider audience the simple mathematical system underlying electroweak theory, which has been making regular appearances in the literature. It may also introduce many readers to the importance of Clifford algebras in particle physics, beyond their implementation in the Dirac equation.

\section{2. $\mathbb{C l}(4)$ in Terms of Ladder Operators}

This construction relies only on the Clifford algebra $\mathbb{C l}(4)$. For the purposes of this discussion, readers may think of $\mathbb{C l}(4)$ simply as the space of $4 \times 4$ complex matrices.

One way to generate $\mathbb{C l}(4)$ is by the set of four matrices,

$$
\begin{array}{rlr}
\gamma_{1} \equiv\left(\begin{array}{cc}
-i \sigma_{x} & 0 \\
0 & i \sigma_{x}
\end{array}\right), & \gamma_{2} \equiv\left(\begin{array}{cc}
i \sigma_{y} & 0 \\
0 & -i \sigma_{y}
\end{array}\right), \\
\gamma_{3} \equiv\left(\begin{array}{cc}
0 & -i \mathbb{I} \\
-i \mathbb{I} & 0
\end{array}\right), & \gamma_{4} \equiv\left(\begin{array}{cc}
0 & \mathbb{I} \\
-\mathbb{I} & 0
\end{array}\right),
\end{array}
$$

where the usual Pauli matrices are given by $\sigma_{x}=\left(\begin{array}{cc}0 & 1 \\ 1 & 0\end{array}\right), \sigma_{y}=\left(\begin{array}{cc}0 & -i \\ i & 0\end{array}\right)$, and $\sigma_{z}=$ $\left(\begin{array}{rr}1 & 0 \\ 0 & -1\end{array}\right)$, while $\mathbb{I}$ represents the $2 \times 2$ identity. These four $\gamma$-matrices obey the relations

$$
\left\{\gamma_{j}, \gamma_{k}\right\} \equiv \gamma_{j} \gamma_{k}+\gamma_{k} \gamma_{j}=-2 \delta_{j k} \mathbb{I}_{4 \times 4}, \quad \forall j, k .
$$

Readers should note that there is indeed no relevant signature for $\mathbb{C l}(4)$, seeing as how the algebra is over the field $\mathbb{C}$.

The Clifford algebra $\mathbb{C l}(4)$ can be built up from products of the four $\gamma_{j}$, as depicted in Fig. 1.

$$
\begin{array}{ccccc}
\gamma_{1} \gamma_{2} \gamma_{3} \gamma_{4} \\
\gamma_{1} \gamma_{2} \gamma_{3} & \ldots & \gamma_{2} \gamma_{3} \gamma_{4} \\
\gamma_{1} \gamma_{2} & \gamma_{1} \gamma_{3} & \ldots & \gamma_{2} \gamma_{4} & \gamma_{3} \gamma_{4} \\
& \gamma_{1} & \gamma_{2} & \gamma_{3} & \gamma_{4}
\end{array}
$$

Fig. 1. Four $\gamma_{j}$ generate the 16-complex-dimensional $\mathbb{C l}(4)$. 
Now, it is straightforward to see that the 4 - $\mathbb{C}$-dimensional generating space, spanned by the $\gamma_{j}$, may be rewritten in terms of a new basis,

$$
\left\{\gamma_{j}\right\} \mapsto\left\{\beta_{1}, \beta_{2}, \beta_{1}^{\dagger}, \beta_{2}^{\dagger}\right\}
$$

where

$$
\beta_{1} \equiv \frac{1}{2}\left(-\gamma_{2}+i \gamma_{1}\right), \quad \beta_{2} \equiv \frac{1}{2}\left(-\gamma_{4}+i \gamma_{3}\right),
$$

and $\beta_{1}^{\dagger}, \beta_{2}^{\dagger}$ are the usual Hermitian conjugates of these. The two $\beta_{j}$ may be viewed as lowering operators, and the $\beta_{j}^{\dagger}$ as raising operators, which obey the following anticommutation relations,

$$
\left\{\beta_{j}, \beta_{k}\right\}=\left\{\beta_{j}^{\dagger}, \beta_{k}^{\dagger}\right\}=0, \quad\left\{\beta_{j}, \beta_{k}^{\dagger}\right\}=\delta_{j k}, \quad \forall j, k=1,2 .
$$

It is then clear that the $16-\mathbb{C}$-dimensional algebra $\mathbb{C l}(4)$ may be rewritten entirely in terms of these four ladder operators.

\section{Ladder Symmetries}

Given a Clifford algebra generated entirely from ladder operators, we would now like to know what symmetries preserve this generating ladder system. To this end, we will then consider only those transformations which satisfy the following properties.

(1) We will be interested in transformations, $G$, on our ladder operators, $\mathcal{O}$, of the form $G \mathcal{O} G^{-1}$. These can easily be seen to preserve the anticommutation relations (5). The group element $G$ will be taken to be $G=e^{-i \phi_{k} g_{k}}$, where $\phi_{k} \in \mathbb{C}$ and $g_{k}$ is allowed to be any element in $\mathbb{C l}(4)$.

(2) Furthermore, we require that the set of lowering operators be closed under these transformations, and likewise for the set of raising operators, as in Fig. 2.

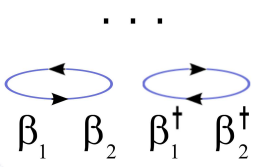

Fig. 2. The Clifford algebra $\mathbb{C l}(4)$ may be rewritten entirely in terms of the ladder operators $\beta_{i}$ and $\beta_{j}^{\dagger}$. We will be interested in transformations under which the lowering operators are closed, and likewise for the raising operators. 
More precisely, we require that for given $b_{i}, b_{i}^{\prime} \in \mathbb{C}$, there exist $c_{j}, c_{j}^{\prime} \in \mathbb{C}$, such that

$$
G b_{i} \beta_{i} G^{-1}=c_{j} \beta_{j}, \quad G b_{i}^{\prime} \beta_{i}^{\dagger} G^{-1}=c_{j}^{\prime} \beta_{j}^{\dagger} .
$$

(3) As a final condition, we will also require that any raising operator and lowering operator, which are the Hermitian conjugates of each other before a transformation are also the Hermitian conjugates of each other after the transformation. In other words, we require that these transformations commute with Hermitian conjugation,

$$
G \beta_{j}^{\dagger} G^{-1}=\left(G^{-1}\right)^{\dagger} \beta_{j}^{\dagger} G^{\dagger} .
$$

Imposing these three conditions on a generic element $\phi_{k} g_{k} \in \mathbb{C l}(4)$ leaves us with only four nontrivial solutions over $\mathbb{R}$ :

$$
\begin{array}{ll}
T_{1} \equiv\left(\begin{array}{rrrr}
0 & 0 & 0 & 0 \\
0 & 0 & 1 & 0 \\
0 & 1 & 0 & 0 \\
0 & 0 & 0 & 0
\end{array}\right), & T_{2} \equiv\left(\begin{array}{rrrr}
0 & 0 & 0 & 0 \\
0 & 0 & -i & 0 \\
0 & i & 0 & 0 \\
0 & 0 & 0 & 0
\end{array}\right), \\
T_{3} \equiv\left(\begin{array}{rrrr}
0 & 0 & 0 & 0 \\
0 & 1 & 0 & 0 \\
0 & 0 & -1 & 0 \\
0 & 0 & 0 & 0
\end{array}\right), \quad N \equiv\left(\begin{array}{llll}
0 & 0 & 0 & 0 \\
0 & 1 & 0 & 0 \\
0 & 0 & 1 & 0 \\
0 & 0 & 0 & 2
\end{array}\right) .
\end{array}
$$

Readers may confirm that the three $T_{i}$ generate $S U(2)$ and that $N$, a $U(1)$ generator, commutes with all three of the $T_{i}$. At the group level, this symmetry may be seen to be $S U(2) \times U(1) / \mathbb{Z}_{2} \simeq U(2)$. Hence, instead of specifying the three conditions above, we could have arrived at this symmetry more expediently by simply stating that we are interested in the unitary symmetries of these ladder operators.

\section{States}

Now that we have identified the $S U(2)$ and $U(1)$ symmetry generators for this Clifford algebraic system, we would like to establish a set of states on which these symmetries act.

$\mathbb{C l}(4)$ has just a single irreducible representation, which acts on a 4 - $\mathbb{C}$ dimensional space of states. The usual way to write this set of states is as a $4-\mathbb{C}$ dimensional column vector. An alternate way to write this set of states is as a special subspace within the $4 \times 4 \mathbb{C}$ matrices.

For various purposes, it will be useful for us to build these states as a special 4 - $\mathbb{C}$ dimensional subspace within the $4 \times 4 \mathbb{C}$ matrices. As we have shown earlier, $\mathbb{C l}(4)$ can be written entirely in terms of ladder operators. Hence, so can any subspace of $\mathbb{C l}(4)$. We will then find that this set of states can be written purely in terms of our ladder operators, $\beta_{i}$ and $\beta_{j}^{\dagger}$. As a result, transformations on the ladder operators can now be seen to induce transformations on the states. 
Now, this special subspace of the Clifford algebra is otherwise known as a minimal one-sided ideal. Incidentally, minimal one-sided ideals of Clifford algebras pro-

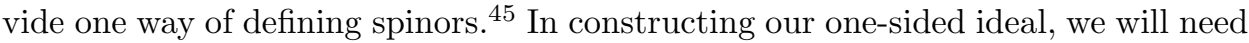
to make the inconsequential choice between building it as a left ideal, or a right ideal. Here, we decide rather arbitrarily to construct a minimal right ideal.

Given an algebra, $A$, a right ideal, $B$, is a subalgebra of $A$ whereby $\underline{b} a$ is in $B$ for all $\underline{b}$ in $B$, and for any $a$ in $A$. That is, no matter which $a$ we multiply onto $\underline{b}$, the new product, $\underline{b}^{\prime} \equiv \underline{b} a$ must be in the subspace $B$. Intuitively, an ideal can be thought of as an algebra's stable subspace, in that it persists, no matter which algebraic element is multiplied onto it. Finally, a minimal right ideal is a right ideal which contains no other right ideals other than $\{0\}$ and itself.

The construction of minimal right ideals within complex Clifford algebras, $\mathbb{C l}(n)$, is quite straightforward. $\frac{5,45}{}$ We begin by building the nilpotent object $\Omega$, which is defined for $\mathbb{C l}(4)$ to be the product of our lowering operators, $\Omega \equiv \beta_{2} \beta_{1}$. From our nilpotent $\Omega$, we construct what we will know as our (formal) vacuum state, $\Omega^{\dagger} \Omega$. The object $\Omega^{\dagger} \Omega$ is a vacuum state only in the formal sense, as it will not represent the zero-particle state. Instead for us, it will represent the sterile right-handed neutrino.

Finally, our space of states is given simply by right-multiplying each and every element of $\mathbb{C l}(4)$ onto the projector $\Omega^{\dagger} \Omega$; that is, $S \equiv \Omega^{\dagger} \Omega \mathbb{C l}(4)$. This leaves us with a 4 - $\mathbb{C}$-dimensional space, $S$, spanned by the states

$$
\left\{\Omega^{\dagger} \Omega, \Omega^{\dagger} \Omega \beta_{1}^{\dagger}, \Omega^{\dagger} \Omega \beta_{2}^{\dagger}, \Omega^{\dagger} \Omega \beta_{1}^{\dagger} \beta_{2}^{\dagger}\right\} .
$$

It is clear that our minimal right ideal then naturally exhibits the structure of a Fock space. ${ }^{\mathrm{a}}$

\section{Leptons Under $s u(2)_{L}$ and $u(1)_{Y}$}

Now that we have constructed our 4 - $\mathbb{C}$-dimensional space of states, we would like to identify which particles these states represent. Of course, this is achieved by characterizing how the states transform under a given symmetry.

In keeping with the transformations of ladder operators, $\beta_{j}^{\prime}=G \beta_{j} G^{-1}$ and $\beta_{j}^{\dagger^{\prime}}=G \beta_{j}^{\dagger} G^{-1}$, our states consequently transform as

$$
S^{\prime}=e^{-i \phi_{k} g_{k}} S e^{i \phi_{k} g_{k}}=S e^{i \phi_{k} g_{k}},
$$

where the $g_{k}$ here are given by Eqs. (8). It so happens that our generators (8) have the property $g_{k} S=0$, which explains the final equality of Eq. (10).

Under the $S U(2)$ generators $T_{i}$, we find that the states $\Omega^{\dagger} \Omega$ and $\Omega^{\dagger} \Omega \beta_{1}^{\dagger} \beta_{2}^{\dagger}$ each behave as singlets, whereas the states $\Omega^{\dagger} \Omega \beta_{1}^{\dagger}$ and $\Omega^{\dagger} \Omega \beta_{2}^{\dagger}$ transform into each other as a doublet. For this reason, we will then identify $\Omega^{\dagger} \Omega$ and $\Omega^{\dagger} \Omega \beta_{1}^{\dagger} \beta_{2}^{\dagger}$ as righthanded states, and $\Omega^{\dagger} \Omega \beta_{1}^{\dagger}$ and $\Omega^{\dagger} \Omega \beta_{2}^{\dagger}$ as left-handed states.

\footnotetext{
${ }^{\text {a } I t ~ m a y ~ b e ~ n o t e d ~ t h a t ~ t h e ~ F o c k ~ s t a t e ~} \Omega^{\dagger} \Omega \beta_{1}^{\dagger} \beta_{2}^{\dagger}$ can also be written more compactly as $\Omega^{\dagger}$.
} 


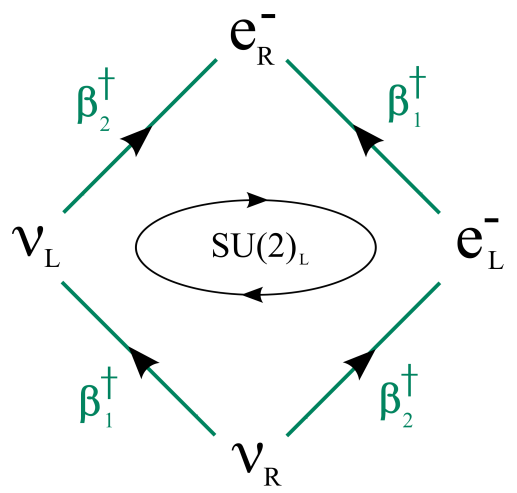

Fig. 3. Fock space structure of leptonic states. These states transform under $s u(2)_{L}$ and $u(1)_{Y}$ : symmetries determined by the ladder operators of the system. It is the hierarchy induced by the $\beta_{j}^{\dagger}$ which allow one chirality to be chosen over another. Readers should note that we did not need to rely on extra Higgs bosons, gauge bosons, nor ad hoc projectors here for $s u(2)$ to act on only a single chirality.

Defining weak hypercharge as $Y \equiv-1 / 2 N$ then assigns to our vacuum state, $\Omega^{\dagger} \Omega$, a hypercharge of zero, to our left-handed states, $\Omega^{\dagger} \Omega \beta_{1}^{\dagger}$ and $\Omega^{\dagger} \Omega \beta_{2}^{\dagger}$, each a hypercharge of $-1 / 2$, and to our final right-handed state, $\Omega^{\dagger} \Omega \beta_{1}^{\dagger} \beta_{2}^{\dagger}$, a hypercharge of -1 . Here, we have defined hypercharge according to the conventions of Schwartz. .48

With their behaviour under the ladder symmetries $s u(2)_{L}$ and $u(1)_{Y}$, we may then identify $\Omega^{\dagger} \Omega$ as a right-handed neutrino, $\Omega^{\dagger} \Omega \beta_{1}^{\dagger}$ as a left-handed neutrino, $\Omega^{\dagger} \Omega \beta_{2}^{\dagger}$ as a left-handed electron, and $\Omega^{\dagger} \Omega \beta_{1}^{\dagger} \beta_{2}^{\dagger}$ as a right-handed electron. Our states then take the matrix form

$$
S=\left(\begin{array}{cccc}
\mathcal{V}_{R} & \mathcal{V}_{L} & \mathcal{E}_{L}^{-} & \mathcal{E}_{R}^{-} \\
0 & 0 & 0 & 0 \\
0 & 0 & 0 & 0 \\
0 & 0 & 0 & 0
\end{array}\right)
$$

where $\mathcal{V}_{R}, \mathcal{V}_{L}, \mathcal{E}_{L}^{-}, \mathcal{E}_{R}^{-}$are complex coefficients (see Fig. 3).

\section{Charge Quantization}

It is a feature of this model that the $U(1)$ generator should be proportional to $N$, the number operator for the system, $N=\sum_{j=1,2} \beta_{j} \beta_{j}^{\dagger}$. Number operators, of course, count the number of raising operator excitations in each state, and must therefore take on only integer values. Hence, we find a straightforward explanation for the quantization of hypercharge $Y$ on leptons.

This same number-operator-quantization was a central feature in the case of electric charge, $Q$, as described in earlier work. ${ }^{5,33}$ There, we examined the Clifford algebra $\mathbb{C l}(6)$ and found ladder symmetries corresponding uniquely to $s u(3)_{c}$ and $u(1)_{\mathrm{em}}$. This time, the ladder symmetries acted on minimal left ideals, which were then found to behave as does a generation of quarks and leptons. 
Readers are encouraged to see the earlier work of Barducci et al. ${ }^{2-4}$ who showed similar Clifford algebraic extensions. Woit also identified further standard model structure in his paper, $\frac{1}{2}$ via (projective) geometrical methods.

\section{Relation to Supermultiplets}

Readers familiar with supersymmetry will notice a striking resemblance between the minimal right ideal constructed here, and the supermultiplets of certain $N=2$ extended SUSY models. However, it bears emphasizing that the irreducible representation constructed here is not inherently supersymmetric. In particular, the ladder operators $\beta_{i}$ and $\beta_{i}^{\dagger}$ do not carry spin indices, and hence do not map bosons to fermions and vice versa.

The 4- $\mathbb{C}$-dimensional $\mathbb{C l}(4)$ irreducible representation constructed for this paper is a special case of the more general $2^{n} \mathbb{C}$-dimensional $\mathbb{C l}(2 n)$ irreducible representation. In the general case, the Clifford algebra's $2 n$-dimensional generating space is partitioned into two maximal totally isotropic subspaces, according to a bilinear form given by the anticommutator. The elements of this MTIS are then used to construct an idempotent (or alternately, nilpotent) object, and subsequently a minimal one-sided ideal. The construction may be most familiar in the case of Spin(10) and $S U(5)$ grand unified theories, where the internal degrees of freedom of quarks and leptons may be described by the irreducible representation of the Clifford algebra $\mathbb{C l}(10)$ (for further details, see Refs. $\underline{5}, \underline{45}-47$ ).

\section{Summary and Outlook}

This review illustrates the importance of ladder structure within particle models, as emphasized by a number of authors. $\underline{1}^{-\underline{5}}$ Here we have demonstrated how the Clifford algebra $\mathbb{C l}(4)$ can be constructed purely from ladder operators. These ladder operators are subject to a unitary symmetry which turns out to be generated by $s u(2)$ and $u(1)$. We identify these symmetries as weak isospin and hypercharge.

These ladder operators furthermore allow us to build a set of states in the form of a Fock space. Under $s u(2)$ and $u(1)$, these states are found to behave as does the set of left- and right-handed electrons and neutrinos. In particular, the $u(1)$ generator automatically assigns those eigenvalues corresponding to weak hypercharge. The $s u(2)$ generators are seen to act nontrivially on only half of the Fock states, which we thereby identify as left-handed. This is achieved without the aid of extra gauge bosons, Higgs bosons, or ad hoc projectors.

Observations like the one detailed here continue to inform ongoing research along the same lines. $., 22,33,49$ In particular, we continue to work towards understanding where this specific Clifford algebraic structure comes from. Of the infinite number of Clifford algebras available, why would nature choose $\mathbb{C l}(4)$ ? And given this Clifford algebra, why would nature choose unitary ladder symmetries over spin groups? Such choices might again seem puzzling and arbitrary, however, we hold to the expectation that nature will hand over these lessons in time. 


\section{Acknowledgments}

A special thanks to B. Allanach, F. Caravelli, G. Fiore, J. Harrison, M. Hughes, A. Kempf, A. Lasenby, C. Tamarit, V. Zatloukal for feedback on this work. This author is grateful for support from the NSERC Postdoctoral Fellowship, the STFC grant ST/L000385/1, and from the Walter Grant Scott Research Fellowship in Physics at Trinity Hall, University of Cambridge.

\section{References}

1. P. Woit, Nucl. Phys. B 303, 329 (1998).

2. A. Barducci, F. Buccella, R. Casalbuoni, L. Lusanna and E. Sorace, Phys. Lett. B 67, 344 (1977).

3. R. Casalbuoni and R. Gatto, Phys. Lett. B 88, 306 (1979).

4. R. Casalbuoni and R. Gatto, Phys. Lett. B 90, 81 (1980).

5. C. Furey, Standard model physics from an algebra?, Ph.D. thesis, University of Waterloo (2015), www.repository.cam.ac.uk/handle/1810/254719.

6. T. D. Lee and C. N. Yang, Phys. Rev. 104, 254 (1956).

7. C. N. Wu, E. Ambler, R. W. Hayward, D. D. Hoppes and R. P. Hudson, Phys. Rev. 105, 1413 (1957).

8. R. P. Hudson, Reversal of the parity conservation law in nuclear physics, in $A$ Century of Excellence in Measurements, Standards, and Technology, ed. D. R. Lide (CRC Press, 2002) [A Chronicle of Selected NBS/NIST Publications, 1901-2000, NIST Special Publication 958].

9. R. L. Garwin, L. M. Lederman and M. Weinrich, Phys. Rev. 105, 1415 (1957).

10. E. Ambler, R. W. Hayward, D. D. Hoppes, R. P. Hudson and C. S. Wu, Phys. Rev. 106, 1361 (1957).

11. J. Pati and A. Salam, Phys. Rev. D 10, 275 (1974).

12. G. Senjanović and R. N. Mohapatra, Phys. Rev. D 12, 1502 (1975).

13. G. Senjanović, Nucl. Phys. B 153, 334 (1979).

14. R. N. Mohapatra and G. Senjanović, Phys. Rev. D 23, 167 (1981).

15. A. Maiezza, M. Nemevsek, F. Nesti and G. Senjanović, Phys. Rev. D 82, 055022 (2010).

16. L. Basso, K. Mimasu and S. Moretti, J. High Energy Phys. 09, 024 (2012).

17. J. Chakrabortty, J. Gluza, R. Sevillano and R. Szafron, J. High Energy Phys. 07, 038 (2012).

18. P.-H. Gu, Phys. Lett. B 713, 485 (2012).

19. W. Dekens and D. Boer, Nucl. Phys. B 889, 727 (2014).

20. S. Bertolini, A. Maiezza and F. Nesti, Phys. Rev. D 89, 095028 (2014).

21. B. A. Dobrescu and Z. Liu, J. High Energy Phys. 10, 118 (2015).

22. C. Furey, $S U(3)_{C} \times S U(2)_{L} \times U(1)_{Y} \times\left(U(1)_{X}\right)$ as a symmetry of division algebraic ladder operators, in preparation.

23. J. S. R. Chisholm and R. S. Farwell, J. Phys. A: Math. Gen. 32, 2805 (1999).

24. C. Doran and A. Lasenby, Geometric Algebra for Physicists (Cambridge University Press, 2003).

25. D. Hestenes, Gauge gravity and electroweak theory, arXiv:0807.0060 [gr-qc].

26. M. Pavšič, Phys. Lett. B 692, 212 (2010).

27. I. Herbut, Phys. Rev. D 87, 085002 (2013).

28. B. P. Dolan and R. J. Szabo, Phys. Rev. D 88, 066002 (2013).

29. R. Abłamowicz, I. Gonçalves and R. da Rocha, J. Math. Phys. 55, 103501 (2014). 
30. C. Furey, J. High Energy Phys. 10, 046 (2014), arXiv:1405.4601 [hep-th].

31. H. Gies and S. Lippoldt, Phys. Lett. B 743, 415 (2015).

32. A. László, A natural extension of the conformal Lorentz group in a field theory context, in Proc. Gribov-85 Memorial Workshop (2015), arXiv:1507.08039 [math-ph].

33. C. Furey, Phys. Lett. B 742, 195 (2015), arXiv:1603.04078 [hep-th].

34. G. E. McClellan, Adv. Appl. Clifford Algebras 27, 761 (2017).

35. T. Misumi, J. High Energy Phys. 12, 063 (2013).

36. C. P. Martín, Phys. Rev. D 89, 065018 (2014).

37. S. J. Gates Jr., T. Hubsch and K. Stiffler, Int. J. Mod. Phys. A 30, 1550042 (2015).

38. A. Connes and J. Lott, Nucl. Phys. B 18, 29 (1990).

39. F. Girelli, Lett. Math. Phys. 57, 7 (2001).

40. A. Connes and M. Marcolli, Noncommutative Geometry, Quantum Fields and Motives (American Mathematical Society, 2007).

41. L. Boyle and S. Farnsworth, New J. Phys. 16, 123027 (2014).

42. F. D'Andrea and L. Dabrowski, The standard model in noncommutative geometry and Morita equivalence, arXiv:1501.00156 [math-ph].

43. J. W. Barrett and L. Glaser, J. Phys. A: Math. Theor. 49, 245001 (2016).

44. A. H. Chamseddine, A. Connes and W. D. van Suijlekom, J. High Energy Phys. 1511, 011 (2015).

45. R. Abłamowicz, Construction of spinors via Witt decomposition and primitive idempotents: A review, in Clifford Algebras and Spinor Structures (Kluwer Academic Publishers, 1995).

46. R. N. Mohapatra, Unification and Supersymmetry: The Frontiers of Quark-Lepton Physics (Springer, 2003).

47. J. Baez and J. Huerta, Bull. Am. Math. Soc. 47, 483 (2010).

48. M. D. Schwartz, Quantum Field Theory and The Standard Model (Cambridge University Press, 2014).

49. P. Woit, Quantum Theory, Groups and Representations (Springer, 2017). 